\title{
SMARCB1/INI1 germline mutations contribute to $10 \%$ of sporadic schwannomatosis
}

\author{
Guillaume Rousseau ${ }^{1,2}$, Tetsuro Noguchi², Violaine Bourdon², Hagay Sobol ${ }^{2,3}$, Sylviane Olschwang ${ }^{2,4^{*}}$
}

\begin{abstract}
Background: Schwannomatosis is a disease characterized by multiple non-vestibular schwannomas. Although biallelic NF2 mutations are found in schwannomas, no germ line event is detected in schwannomatosis patients. In contrast, germline mutations of the SMARCB1 (IN/1) tumor suppressor gene were described in familial and sporadic schwannomatosis patients.

Methods: To delineate the SMARCB1 gene contribution, the nine coding exons were sequenced in a series of 56 patients affected with a variable number of non-vestibular schwannomas.

Results: Nine variants scattered along the sequence of SMARCB1 were identified. Five of them were classified as deleterious. All five patients carrying a SMARCB1 mutation had more multiple schwannomas, corresponding to $10.2 \%$ of patients with schwannomatosis. They were also diagnosed before 35 years of age.

Conclusions: These results suggest that patients with schwannomas have a significant probability of carrying a SMARCB1 mutation. Combined with data available from other studies, they confirm the clinical indications for genetic screening of the SMARCB1 gene.
\end{abstract}

\section{Background}

Neurofibromatoses (NF) are an heterogeneous group of genetic disorders predisposing to various tumors of the nervous system, divided into two well recognized distinct clinical entities, NF1 and NF2. Patients with multiple nonvestibular schwannomas have been assembled into a particular category within neurofibromatoses called schwannomatosis [1]. The SMARCB1 gene has been found to harbor germline alterations in both familial and sporadic schwannomatosis patients $[2,3]$, with a greater number of spinal schwannomas in familial cases and the presence of meningiomas [4] although this latter point remains debated [5]. To improve the clinical indications for SMARCB1 molecular screening in medical genetics practice, we evaluated its implication in a series of patients exhibiting non-vestibular schwannomas and no NF2 germline alteration.

\section{Methods}

From 1992 to 2006, 303 patients were referred from the French outpatient genetic clinics for point mutations

\footnotetext{
* Correspondence: sylviane.olschwang@inserm.fr

${ }^{2}$ Laboratory of Molecular Oncogenetics, Institut Paoli-Calmettes; Marseille, France

Full list of author information is available at the end of the article
}

and genomic rearrangements analysis of the NF2 gene to investigate the presence of schwannomas. All patients or legal representatives signed an informed consent for genetic analyses related to their disease according to the French law (Public Health Code, article R145-15-4). No specific consent was required for the present study, as the SMARB1 analysis is part of the genetic tests proposed to patients affected with schwannomas. Analyses were performed in a laboratory labeled by the French Biomedicine Agency ABM (lab no. 2008-gen-01). A subgroup of 56 cases exhibiting non-vestibular schwannomas as confirmed by MRI examination at time of diagnosis were selected for SMARCB1 point mutations screening. Medical reports were collected (number and localization of schwannomas, age at diagnosis and family history). Exons and splicing junctions of the SMARCB1 gene (NM_003073, NG_009303.1) were analyzed by sequencing using the SeqScape software (Applied Biosystems, Courtaboeuf France) after PCR amplification (Table 1). Functional consequence of intronic variants was evaluated using NetGene2, GeneSplicer and a splice-site model that accounts for adjacent and nonadjacent pair wise dependencies MaxEnt.

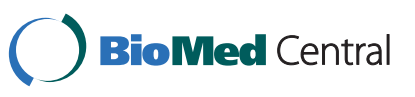




\begin{tabular}{|c|c|}
\hline Exon & Primer pair \\
\hline 1 & 5'CCCTCCTGATCCCTCGCAGC/5'CGGGCTACCTCGGAGCCGAT \\
\hline 2 & 5'CTGCGACCCTTATAATGAGC/5'GCGAGTGGTTTTGAAACAGG \\
\hline 3 & 5'ACCAGCAGAGTGACCCAGTG/5'AGAGATGCCCTGGCCAGGAA \\
\hline 4 & 5'GGATCAGGTCCTATACTGAC/5'AACTAAGGCGGAATCAGCAC \\
\hline 5 & 5'TGCATACCTAGGGCTCCGG/5'GCCCGACTGCCTTGTACCAT \\
\hline 6 & 5TGGTGCAATCTCTTGGCATC/5TCAGTGCTCCATGATGACAC \\
\hline 7 & 5'TGGGCTGCAAAAGCTCTAAC/5'CGCTCACACAGAGAAGTCT \\
\hline 8 & 5'ATCCACTGGGTGCCAGCAGT/5TCTGCCTGGAAAGCCAGGTG \\
\hline 9 & 5'CCCTGTAGAGCCTTGGGAAG/5'GCCTCTGTCCTTGCCAGAAG \\
\hline
\end{tabular}

\section{Results}

Thirty-one females and 25 males were included in the study. The age of onset ranged from 2 to 70 years. Six patients had a single schwannoma, 11 patients had 2 to 7 and 39 patients more than 10 schwannomas. Two patients had a positive family history. Nine different sequence variants scattered along the sequence of SMARCB1 were found (Table 2). Three were present in 12 patients, 10 in a heterozygous and two in a homozygous state indicating a complete linkage disequilibrium (Fisher exact test, $\mathrm{p}<10^{-6}$ ). Two of them were present in dbSNP as rs2229354 (c.897G > A) and rs5030613 (c.1119-41G > A); the third change (c.1149-41G > A) was also considered as a polymorphism. The remaining 6 variants were unique: c.30del, c. $34 \mathrm{C}>\mathrm{T}$ and c.832C > $\mathrm{T}$ were expected to truncate the protein; c.500+5G $>\mathrm{T}$ was predicted to suppress the donor splicing site of exon 4, leading to p.Arg121_Cys167delinsSer and lacking the DNA-binding domain. Using the MaxEnt program, maximum entropy of -5.62 was computed compared to 0.87 with the normal sequence. Considering the existence of a rare long isoform, as a 51-bp extension at the 3 ' end of exon 4 , this variant also encoded c.505G > T was expected to produce a $\mathrm{p}$. Gly169X truncated protein lacking the two highly conserved regions Rpt1 and Rpt2 and the coiled-coil domain. The variant c.971_978delinsTGCTACCT implicated the highly conserved $\mathrm{Lys}_{324}$ and $\mathrm{Tyr}_{326}$ aminoacids, $\mathrm{Tyr}_{326}$ being involved in the coiled-coil domain and possibly in the nuclear localization signal and/or the binding of Akt. All five were considered deleterious. The transversion c.501-23T > G was not predicted to affect splicing using NetGene2 and GeneSplicer and was classified as not responsible for the disease. All five patients carrying a SMARCB1 mutation were sporadic patients diagnosed before 35 years of age with multiple non-vestibular schwannomas.

\section{Discussion and conclusions}

Five mutations were identified through the analysis of the SMARCB1 gene for point mutations in a series of 56 NF2-negative patients affected with non-vestibular schwannomas. Patients carrying a mutation presented with multiple schwannomas at a young age, i.e. $10.2 \%$ in schwannomatosis patients [6] and 20\% of patients diagnosed before 35 years of age, none with any family history of schwannomas. This proportion remains lower than that described in familial schwannomatosis $[3,7,8]$; however neither somatic mosaicisms nor genomic rearrangements were investigated $[2,7]$. Tumor samples were not available thus precluding dosage analyses and the demonstration of SMARCB1 and/or NF2 somatic inactivation [3].

Schwannomatosis is a rare condition characterized by the development of multiple schwannomas and the lack of vestibular nerve involvement. As germline SMARCB1 mutations have never been reported in cases of vestibular schwannomas, the presence of multiple non-vestibular schwannomas appears a good criterion to first investigate the SMARCB1 gene, as reported through several studies (Table 3). From our study, as mutations were found in patients diagnosed before 35 years of age, this aspect might also be considered to enlarge the SMARCB1 analysis first to the search for genomic rearrangements and somatic mosaicisms and second to the NF2 gene screening. On the other hand, the presence of

Table 2 Point variations and phenotypic description of the patients exhibiting a SMARCB1 variation

\begin{tabular}{|c|c|c|c|c|c|c|}
\hline Patient & Exon & Nucleotide change & Expected effect & Sex & Age & Disease \\
\hline N00390 & 1 & c.30del & p.Phe10LeufsX6 & $\mathrm{F}$ & 35 & 11 non-vestibular schwannomas \\
\hline N00837 & 1 & c.34C > T & p.Gln12X & $\mathrm{F}$ & 24 & Multiple spinal schwannomas \\
\hline \multirow[t]{3}{*}{ N00328 } & 4 (ivs) & c. $[500+5 G>T, 505 G>T]$ & p.[Arg121_Cys167delinsSer,Gly169X] & M & 27 & 1 facial nerve \\
\hline & & & & & & 7 spinal schwannomas \\
\hline & & & & & & 2 meningiomas \\
\hline N00106 & 5 (ivs) & c.501-23T > G & - & M & 56 & 2 spinal schwannomas \\
\hline N00225 & 7 & c.832C > T & p.Gln278X & M & 22 & Multiple schwannomas and meningiomas \\
\hline N00334 & 7 & c.971_978delinsTGCTACCT & p.Lys324_Tyr326delinslleLeuPro & M & 30 & Unilateral V-, VII-, spinal schwannomas \\
\hline 12 cases & & $\begin{array}{l}\text { c.[897G > A;986+57_986+ } \\
\text { 58dup;1119-41G > A] }\end{array}$ & & & & \\
\hline
\end{tabular}


Table 3 Review of the clinical description of SMARCB1 mutation patients affected with non-rhabdoid tumors

\begin{tabular}{lll}
\hline Reference & Clinical context & Observation \\
\hline$[2]$ & 1 familial schwannomatosis & case report, initial description \\
{$[3]$} & 28 sporadic +15 familial non-vestibular schwannomatosis patients & $2 / 28+5 / 15$ mutations \\
{$[4]$} & 1 familial schwannomatosis with multiple meningiomas & case report \\
{$[5]$} & 47 NF2-negative patients with multiple meningiomas & $0 / 47$ \\
{$[9]$} & 21 sporadic schwannomatosis patients & $1 / 21$ mutation \\
{$[10]$} & 1 familial meningiomatosis & case report \\
\hline
\end{tabular}

vestibular lesions remains the first indication for an $N F 2$ gene molecular analysis.

\section{Acknowledgements}

This work was supported by the French national cancer institute INCa through a grant dedicated to the PHRATries network (Prédispositions Héréditaires Rares Aux Tumeurs, Réseau d'Identification Et de Soins). We are grateful to the French clinicians who faithfully entrust our institution with molecular genetics tests of neurofibromatoses.

\section{Author details}

${ }^{1}$ Neuropediatrics Department, Children Hospital, Montreal, Canada. ${ }^{2}$ Laboratory of Molecular Oncogenetics, Institut Paoli-Calmettes; Marseille, France. ${ }^{3}$ Université La Méditerranée; Marseille, France. ${ }^{4}$ Centre de Recherche en Cancérologie de Marseille, Institut Paoli-Calmettes, 232 boulevard SainteMarguerite, 13009 Marseille, France.

\section{Authors' contributions}

GR has set up the technique for SMARCB1 gene screening. TN and VB have supervised the work, helping in sequencing data interpretation. HS is the head of the laboratory. SO organized patients' recruitment and managed the integrated laboratory approach. All authors read and approved the final manuscript.

\section{Competing interests}

The authors declare that they have no competing interests.

Received: 7 October 2010 Accepted: 24 January 2011

Published: 24 January 2011

\section{References}

1. Lu-Emerson C, Plotkin SR: The neurofibromatoses. Part 2: NF2 and schwannomatosis. Rev Neurol Dis 2009, 6:E81-86.

2. Hulsebos TJ, Plomp AS, Wolterman RA, Robanus-Maandag EC, Baas F, Wesseling P: Germline mutation of INI1/SMARCB1 in familial Schwannomatosis. Am J Hum Genet 2007, 80:805-810.

3. Hadfield KD, Newman WG, Bowers NL, Wallace A, Bolger C, Colley A, McCann E, Trump D, Prescott T, Evans DG: Molecular characterisation of SMARCB1 and NF2 in familial and sporadic schwannomatosis. J Med Genet 2008, 45:332-339.

4. Bacci C, Sestini R, Provenzano A, Paganini I, Mancini I, Porfirio B, Vivarelli R, Genuardi M, Papi L: Schwannomatosis associated with multiple meningiomas due to a familial SMARCB1 mutation. Neurogenetics 2010 11:73-80.

5. Hadfield KD, Smith MJ, Trump D, Newman WG, Evans DG: SMARCB1 mutations are not a common cause of multiple meningiomas. J Med Genet 2010, 47:567-568.

6. MacCollin M, Chiocca EA, Evans DG, Friedman JM, Horvitz R, Jaramillo D, Lev M, Mautner VF, Niimura M, Plotkin SR, Sang CN, StemmerRachamimov A, Roach ES: Diagnostic criteria for schwannomatosis. Neurology 2005, 64:1838-1845.

7. Boyd C, Smith MJ, Kluwe L, Balogh A, Maccollin M, Plotkin SR: Alterations in the SMARCB1 (INI1) tumor suppressor gene in familial schwannomatosis. Clin Genet 2008, 74:358-366.
8. Hulsebos TJ, Kenter SB, Jakobs ME, Baas F, Chong B, Delatycki MB SMARCB1/INI1 maternal germline mosaicism in schwannomatosis. Clin Genet 2010, 77:86-91.

9. Sestini R, Bacci C, Provenzano A, Genuardi M, Papi L: Evidence of a four-hit mechanism involving SMARCB1 and NF2 in schwannomatosis-associated schwannomas. Hum Mutat 2008, 29:227-231.

10. Christiaans I, Kenter SB, Brink HC, van Os TA, Baas F, van den Munckhof $P$, Kidd AM, Hulsebos TJ: Germline SMARCB1 mutation and somatic NF2 mutations in familial multiple meningiomas. J Med Genet 2010.

11. Smith MJ, Boyd CD, MacCollin MM, Plotkin SR: Identity analysis of schwannomatosis kindreds with recurrent constitutional SMARCB1 (INI1) alterations. Clin Genet 2009, 75:501-502

\section{Pre-publication history}

The pre-publication history for this paper can be accessed here: http://www.biomedcentral.com/1471-2377/11/9/prepub

doi:10.1186/1471-2377-11-9

Cite this article as: Rousseau et al: SMARCB1/INI1 germline mutations contribute to $10 \%$ of sporadic schwannomatosis. BMC Neurology 2011 $11: 9$

\section{Submit your next manuscript to BioMed Central and take full advantage of:}

- Convenient online submission

- Thorough peer review

- No space constraints or color figure charges

- Immediate publication on acceptance

- Inclusion in PubMed, CAS, Scopus and Google Scholar

- Research which is freely available for redistribution

Submit your manuscript at www.biomedcentral.com/submit
C Biomed Central 